\title{
An observation on the regioselectivity of ring-opening of some substituted cyclohexenyl epoxides
}

\author{
Madeleine Helliwell, Eric J. Thomas,* and Clare Vickers \\ The School of Chemistry, The University of Manchester, Manchester, M13 9PL, UK \\ E-mail: e.j.thomas@manchester.ac.uk
}

Dedicated to Professor Atta-ur-Rahman on the occasion of his $65^{\text {th }}$ birthday

\begin{abstract}
Attempted epoxidation of the cyclohexenyl carboxylic acid 11 using $m$-chloroperoxybenzoic acid was accompanied by epoxide ring-opening and gave a mixture of lactones 13, 14 and 15, ratio $20: 40: 40$, respectively. In contrast, treatment of the cis-hydroxyepoxide 18 of the methyl ester 12 with aqueous sulfuric acid in acetonitrile gave the lactone 14 with excellent regioselectivity together with the triol 22. The trans-hydroxyepoxide 19 gave only the lactone 14 under these conditions.
\end{abstract}

Keywords: Epoxide ring-opening, lactone, regioselectivity, abyssomicin C

\section{Introduction}

The abyssomicins are a small group of antibacterial macrocyclic polyketides isolated from a marine Verrucosispora strain which exhibit biological activity as inhibitors of $p$-aminobenzoic acid biosynthesis. ${ }^{1}$ Several approaches to the total synthesis of these compounds have been reported $^{2}$ and two total syntheses have already been described. ${ }^{3}$ We independently ${ }^{4}$ envisaged a strategy for the synthesis of abyssomicin C 1 in which the C8-C9 double-bond would be introduced by ring-closing metathesis, and the metathesis precursor 2 prepared by fusing the tetronic acid fragment onto the bicyclic lactone 3 . This lactone, in turn, was to be prepared by regioselective ring-opening of epoxides 4 . This approach to the tricyclic core of the abyssomicins

differs from those already reported ${ }^{2 \mathrm{a}, 2 \mathrm{~b}, 3 \mathrm{~b}}$ where preformed tetronic acids act as the nucleophile for intramolecular epoxide ring-opening. Since our approach is dependent on the ring-opening of epoxides 4 being regioselective, we report preliminary studies on the regioselectivity of ringopening of these epoxides which were carried out in order to evaluate routes to lactones 3 . 

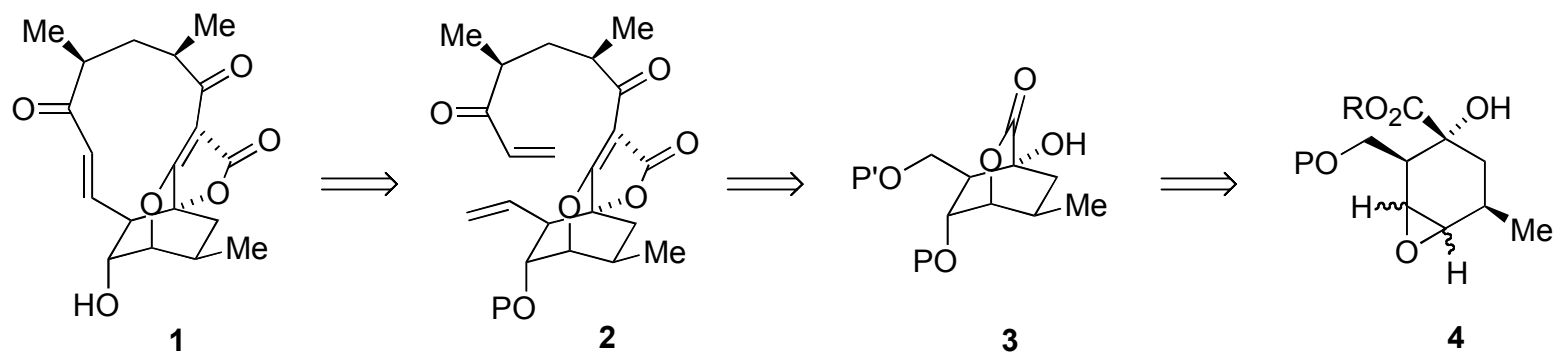

\section{Results and Discussion}

The bicyclic lactone 8 was identified as a precursor of epoxides $\mathbf{4}$. It has been prepared by an intramolecular Diels Alder reaction of sorbyl acrylate ${ }^{5}$ and by an intermolecular Diels Alder reaction of methyl acrylate and sorbyl alcohol mediated by methylmagnesium bromide. ${ }^{6}$ In our hands, however, decarboxylation of the Diels Alder product $5^{7}$ of sorbyl alcohol and maleic anhydride by tributyltin hydride mediated decarbonylation ${ }^{8}$ of the phenylselanyl esters 6 and 7 , was convenient and scaleable, see Scheme 1. Hydroxylation was achieved using the Davis oxaziridine ${ }^{9}$ to give the hydroxy-lactone $9,^{2 b}$ and treatment with potassium hydroxide in toluene in the presence of benzyl bromide gave the benzyl ester $10 .{ }^{2 b, 10}$ This was saponified to give the acid 11 and the acid converted into the methyl ester 12 using trimethylsilyl diazomethane.

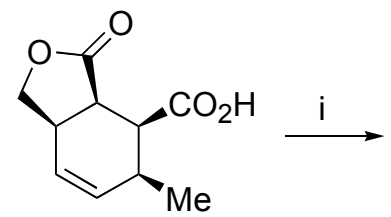

5

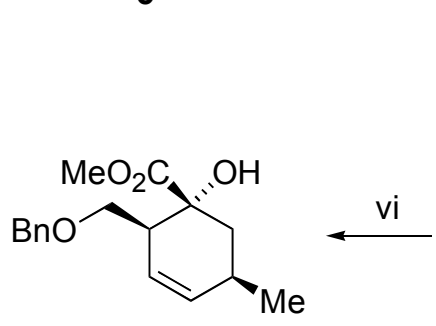

12

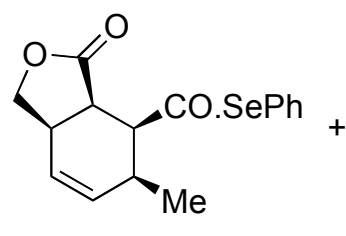

6

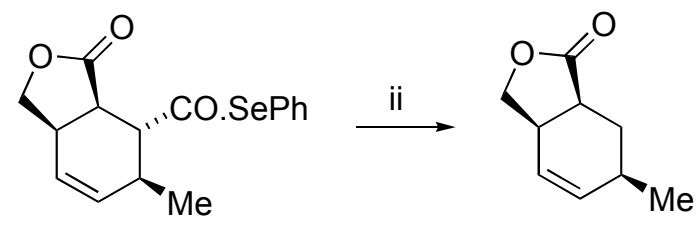

7

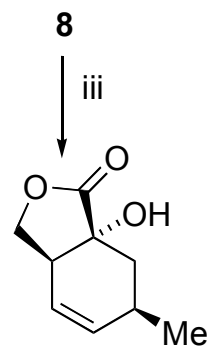

9

Scheme 1. Reagents and conditions: i, (a) $\mathrm{Et}_{3} \mathrm{~N}$, (b) $\mathrm{Bu}_{3} \mathrm{P}, \mathrm{PhSeCl}$, THF, r.t. (6, 60\%; 7, 20\%); ii, $\mathrm{Bu}_{3} \mathrm{SnH}$, AIBN (cat.) (95\%); iii, KHMDS, -78 to $-20{ }^{\circ} \mathrm{C}, 30 \mathrm{~min}$, then ( \pm )-3-phenyl-2(phenylsulfonyl)oxaziridine, $-78{ }^{\circ} \mathrm{C}, 3 \mathrm{~h}$ (70\%); iv, $\mathrm{KOH}, \mathrm{BnBr}$, toluene, reflux, $48 \mathrm{~h}(83 \%)$; $\mathrm{NaOH}, \mathrm{MeCN}$ (59\%); vi, $\mathrm{TMSCHN}_{2}$, toluene, methanol, $0{ }^{\circ} \mathrm{C}, 1 \mathrm{~h}(90 \%)$.

Having prepared esters 10 and 12, and the acid 11, the stereoselectivity of epoxidation of these compounds and the regioselectivity of epoxide ring-opening was investigated. First, a one- 
step procedure of epoxidation and ring-opening was attempted on the acid 11. After treatment of the acid 11 with $m$-chloroperoxybenzoic acid at room temperature in chloroform for $72 \mathrm{~h}$, three products were isolated which were identified, in increasing order of polarity (tlc), as lactone $\mathbf{1 3}$ $(15 \%)$, the required lactone $14(30 \%)$ and the regioisomeric lactone $15(30 \%)$.

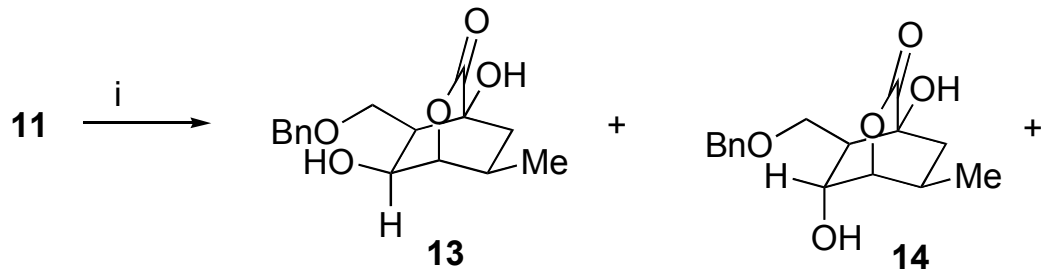

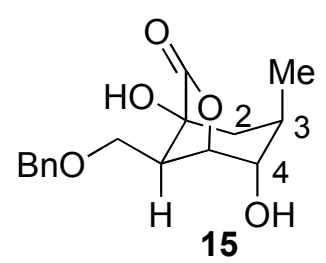
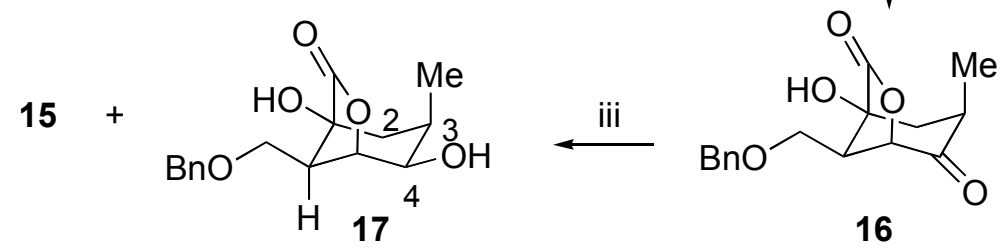

Scheme 2. Reagents and conditions: i, $m \mathrm{CPBA}, \mathrm{CHCl}_{3}$, r.t., $72 \mathrm{~h}(\mathbf{1 3}, 15 \% ; 14,30 \% ; 15,30 \%)$; ii, $\mathrm{PCC}, \mathrm{CH}_{2} \mathrm{Cl}_{2}$ (85\%); iii, $\mathrm{NaBH}_{4}, \mathrm{EtOH}(81 \%, 17: 15=50: 50)$.

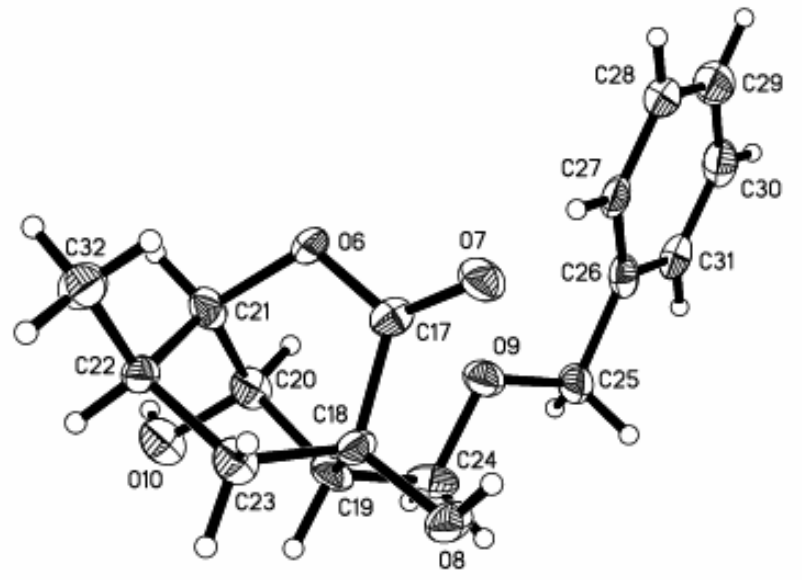

Figure 1. The structure of the lactone $\mathbf{1 4}$ as established by X-ray diffraction.

The structure of the crystalline lactone, which was the second product off the column, was identified as the required lactone 14 by X-ray diffraction, see Figure 1. The most polar lactone was shown to be the regioisomeric lactone $\mathbf{1 5}$ by oxidation to the ketone $\mathbf{1 6}$ followed by reduction which gave a mixture of the separable lactones 15 and 17. Spin-decoupling experiments on lactone 17 indicated that the $\mathrm{CHOH}$ proton was coupled with the $\mathrm{CHCH}_{3}$ proton so confirming the regiochemistry of lactones 15 and 17 as shown. The configuration of the 
hydroxyl bearing carbon, C-4, in lactones 15 and 17 was provisionally assigned on the assumption that the hydroxyl group would be introduced trans with respect to the lactone bridge by epoxide opening. However, this assignment is tentative since the products may be the result of thermodynamic control. The structure of lactone $\mathbf{1 3}$ was assigned by comparison of the carbonyl stretching frequency in its IR with that of lactone 14, from its ${ }^{1} \mathrm{H} N M R$, and from the identification of lactones 14, 15 and 17 as the other three possible regio- and stereo-isomers.

To see whether a regioselective synthesis of the lactone 14 could be achieved, rearrangements of the epoxides obtained from the ester 12 were investigated. Epoxidation of this ester using $m$-chloroperoxybenzoic acid gave a $25: 75$ mixture of the cis-hydroxyepoxide 18 and its trans-diastereoisomer $19(82 \%)$ whereas epoxidation using tert-butyl hydroperoxide in the presence of vanadyl acetylacetonate gave the cis-isomer $18(81 \%)$ only. The structures of epoxides 18 and 19 were assigned by analogy with the literature, ${ }^{2 b}$ and were consistent with nOe studies since enhancement of an epoxide proton was observed on irradiation of the 5- $\mathrm{CH}_{3}$ group only for the cis-epoxide 18 and irradiation of H-2 and H-5 enhanced the epoxide protons only for the trans-isomer 19. The benzyl ester 10 was also epoxidised using $m$-chloroperoxybenzoic acid to give a $33: 67$ mixture of the cis- and trans-hydroxyepoxides $\mathbf{2 0}$ and $\mathbf{2 1}$, the stereochemistry of these epoxides being assigned by analogy with the literature ${ }^{2 b}$ and by comparison with methyl esters 18 and 19.

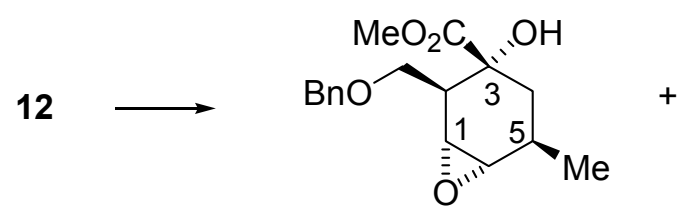

18

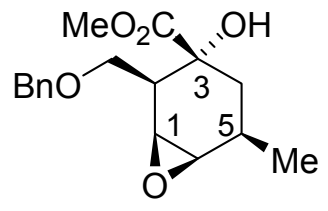

19

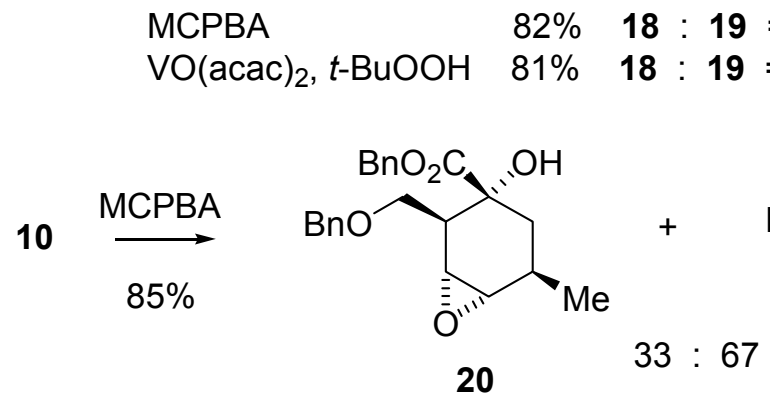

20

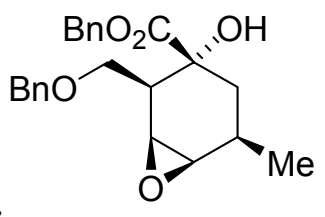

21

Acid catalysed rearrangement of the cis-hydroxyepoxide $\mathbf{1 8}$ using aqueous sulfuric acid in acetonitrile gave the required bicyclic lactone 14 (61\%) together with the trihydroxy-ester 22 (33\%). Under these conditions, the trans-epoxide 19 gave only the lactone 14 (88\%), and the mixture of benzyl ester epoxides 20 and 21 gave the lactone $14(70 \%)$ and the trihydroxy-ester $23(30 \%)$. The configuration of the triol $\mathbf{2 3}$ was assigned on the basis of the diaxial couplings of ca. $10 \mathrm{~Hz}$ observed between $\mathrm{H}-3$ and $\mathrm{H}-4$ and between $\mathrm{H}-4$ and $\mathrm{H}-5$, and the axial - equatorial coupling of $3 \mathrm{~Hz}$ observed between H-2 and H-3. The structure of the trihydroxy methyl ester 22 was assigned by analogy. 


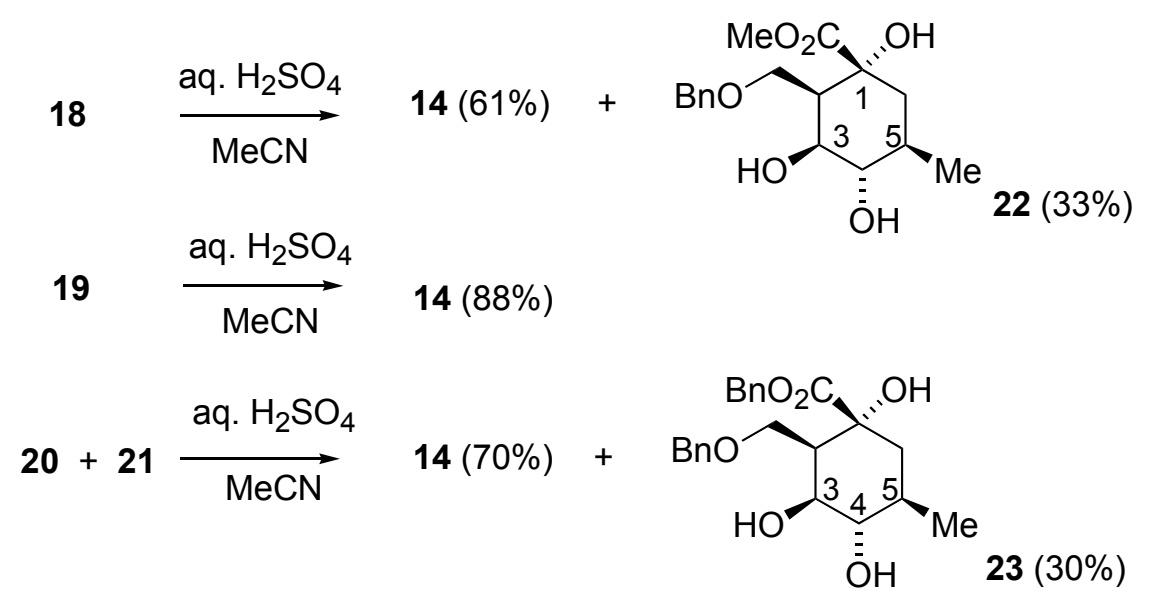

\section{Conclusions}

Since the ester and the epoxide are cis-disposed in the trans-hydroxyepoxide 19, direct participation of the ester in epoxide ring-opening is not possible and so the epoxide ring-opening must involve a regioselective acid catalysed attack of water at $\mathrm{C}-1$ (epoxide numbering), with subsequent lactonisation giving lactone 14. For the cis-hydroxyepoxide 18, the triol 22 must also have been formed by ring-opening of the epoxide initiated by attack of water at $\mathrm{C}-1$. However, the mechanism of formation of the lactone $\mathbf{1 4}$ from the cis-hydroxyepoxide $\mathbf{1 8}$ is ambiguous since either hydrolysis of the epoxide followed by lactonisation or direct acid-catalysed epoxide ring-opening involving the ester carbonyl oxygen may be involved. Similar processes must be involved in the fomation of the lactones 13, 14 and 15 during the reaction between the acid 11 and $m$-chloroperoxybenzoic acid with a double-inversion process giving the unexpected hydroxylactone $\mathbf{1 3}$ in which the hydroxyl group is cis-disposed with respect to the lactone bridge. However, notwithstanding mechanistic complexities, this work has resulted in a regioand stereo-selective synthesis of lactone $\mathbf{1 4}$ which may be useful for a synthesis of the abyssomicins.

\section{Experimental Section}

General Procedures. Low resolution mass spectra were recorded on a Micromass Trio 200 spectrometer and high resolution mass spectra on a Kratos Concept IS spectrometer using electron impact ionisation (EI), chemical ionisation using ammonia (CI) or electrospray in the positive mode (ES). For selenium containing compounds, only peaks corresponding to isotope

${ }^{80} \mathrm{Se}$ are quoted although characteristic isotope patterns were seen. Infrared spectra were recorded on a Genesis FTIR as evaporated films on sodium chloride plates and nuclear magnetic 
resonance (NMR) spectra using deuterated chloroform as solvent unless otherwise stated. Proton NMR spectra were recorded on Bruker $(500 \mathrm{MHz})$ and Varian Unity 500 (500 MHz) spectrometers. Residual non-deuterated solvent was used as internal standar and coupling constants are in Hz. Flash column chromatography was carried out using silica gel $60 \mathrm{H}(40-60$ $\mathrm{nm}, 230-300$ mesh) from Merck. Light Petroleum refers to the fraction that boils between $40{ }^{\circ} \mathrm{C}$ and $60{ }^{\circ} \mathrm{C}$ and was distilled prior to use. Ether refers to diethyl ether which was used without purification.

$(1 R S, 2 S R, 3 S R, 6 R S)$-3-Methyl-9-oxo-8-oxabicyclo[4.3.0]non-4-ene-2-carboselenoic acid Sephenyl ester (6) and (1RS,2RS,3SR,6RS)-3-methyl-9-0xo-8-oxabicyclo[4.3.0]non-4-ene-2carboselenoic acid Se-phenyl ester (7). A solution of triethylamine $(4.74 \mathrm{~mL}, 34 \mathrm{mmol})$ in $\mathrm{CH}_{2} \mathrm{Cl}_{2}(55 \mathrm{~mL})$ was added to a suspension of the acid $\mathbf{5}^{7}(6.68 \mathrm{~g}, 34 \mathrm{mmol})$, in $\mathrm{CH}_{2} \mathrm{Cl}_{2}(165 \mathrm{~mL})$ at r.t under $\mathrm{N}_{2}$ and the mixture stirred at room temperature for $10 \mathrm{~min}$ before being concentrated under reduced pressure to give the triethylammonium carboxylate salt as an orange oil. Tributylphosphine $(12.63 \mathrm{~mL}, 51.17 \mathrm{mmol})$ was separately added dropwise to phenyl selenenyl chloride $(10 \mathrm{~g}, 51.17 \mathrm{mmol})$ in tetrahydrofuran $(250 \mathrm{~mL})$ under $\mathrm{N}_{2}$ and the resulting yellow solution stirred for $15 \mathrm{~min}$. The triethylammonium carboxylate salt was then added as a suspension in tetrahydrofuran $(250 \mathrm{~mL})$. The cloudy mixture was stirred at ambient temperature under $\mathrm{N}_{2}$, for $48 \mathrm{~h}$, added to ether $(300 \mathrm{~mL})$, and the mixture washed with water $(2 \times 500 \mathrm{~mL})$ and dried $\left(\mathrm{MgSO}_{4}\right)$. After concentration under reduced pressure, the orange residue was chromatographed (1:1, ether: light petroleum) to give title compound $7(2.27 \mathrm{~g}, 6.76 \mathrm{mmol}, 20$ \%) as an oil (Found: $\mathrm{M}^{+}+\mathrm{NH}_{4}, 354.0605 . \mathrm{C}_{16} \mathrm{H}_{20} \mathrm{NO}_{3}{ }^{80}$ Se requires $M, 354.0603$ ); $\mathrm{v}_{\max } 1771$, 1708, 1153, 1016, 986 and $739 \mathrm{~cm}^{-1} ; \delta_{\mathrm{H}}\left(500 \mathrm{MHz}, \mathrm{CDCl}_{3}\right) 7.45(2 \mathrm{H}, \mathrm{m}, \mathrm{ArH}), 7.32(3 \mathrm{H}, \mathrm{m}$, $\operatorname{ArH}), 5.71(1 \mathrm{H}$, ddd, $J 2,4,10,4-\mathrm{H}), 5.51(1 \mathrm{H}, \mathrm{td}, J 2.5,10,5-\mathrm{H}), 4.35(1 \mathrm{H}, \mathrm{dd}, J 7,9,7-\mathrm{H})$, $3.97\left(1 \mathrm{H}, \mathrm{dd}, J 4,9,7-\mathrm{H}^{\prime}\right), 3.13(1 \mathrm{H}, \mathrm{m}, 6-\mathrm{H}), 3.12(1 \mathrm{H}, \mathrm{m}, 1-\mathrm{H}), 3.07(1 \mathrm{H}, \mathrm{dd}, J 5.5,8,2-\mathrm{H})$, $2.60(1 \mathrm{H}, \mathrm{m}, 3-\mathrm{H})$ and $1.12\left(3 \mathrm{H}, \mathrm{d}, J 7,3-\mathrm{CH}_{3}\right) ; \delta_{\mathrm{C}}\left(75 \mathrm{MHz}, \mathrm{CDCl}_{3}\right), 202.6,177.0,136.3$, 133.8, 129.9, 129.6, 126.4, 124.2, 72.0, 55.2, 39.8, 34.5, 31.8 and 21.4; Further elution gave the the title compound $6(6.89 \mathrm{~g}, 20.5 \mathrm{mmol}, 60 \%)$ as a white solid, m.p : $88.6-91.4{ }^{\circ} \mathrm{C}$ (Found: $\mathrm{C}$, 57.5; $\mathrm{H}, 4.8 \% ; \mathrm{C}_{16} \mathrm{H}_{16} \mathrm{O}_{3} \mathrm{Se}$ requires $\mathrm{C}, 57.3, \mathrm{H}, 4.8 \%$; Found: $\mathrm{M}^{+}+\mathrm{NH}_{4}, 354.0605$. $\mathrm{C}_{16} \mathrm{H}_{20} \mathrm{NO}_{3}{ }^{80} \mathrm{Se}$ requires $\left.M, 354.0603\right) ; \mathrm{v}_{\max } 1773,1710,1477,1373,1209,1153,1016,986,806$ and $740 \mathrm{~cm}^{-1} ; \delta_{\mathrm{H}}\left(500 \mathrm{MHz}, \mathrm{CDCl}_{3}\right) 7.45(2 \mathrm{H}, \mathrm{m}, \mathrm{ArH}), 7.28(3 \mathrm{H}, \mathrm{m}, \mathrm{ArH}), 5.70(1 \mathrm{H}, \mathrm{ddd}, J$ 2, 4, 12, 4-H), $5.54(1 \mathrm{H}, \mathrm{td}, J 2,12,5-\mathrm{H}), 4.34(1 \mathrm{H}, \mathrm{t}, J 8.5,7-\mathrm{H}), 4.07(1 \mathrm{H}, \mathrm{dd}, J$ 5, 8.5, 7-H'), $3.37(1 \mathrm{H}, \mathrm{t}, J 5.5,2-\mathrm{H}), 3.21(1 \mathrm{H}, \mathrm{dd}, J 5.5,9.5,1-\mathrm{H}), 3.15(1 \mathrm{H}, \mathrm{m}, 6-\mathrm{H}), 2.69(1 \mathrm{H}, \mathrm{m}, 3-\mathrm{H})$, $1.22\left(3 \mathrm{H}, \mathrm{d}, J 7.5,3-\mathrm{CH}_{3}\right) ; \delta_{\mathrm{C}}\left(75 \mathrm{MHz}, \mathrm{CDCl}_{3}\right), 200.3,176.7,136.4,133.6,131.9,129.8,125.0$, $124.9,71.6,54.3,39.9,35.8,31.5$ and $18.4 ; \mathrm{m} / z\left(\mathrm{CI}^{+}\right) 354\left(\mathrm{M}^{+}+18,100 \%\right)$.

$(1 S R, 3 R S, 6 R S)$-3-Methyl-9-oxo-8-oxabicyclo[4.3.0]non-4-ene (8). ${ }^{2 \mathrm{~b}, 5}$ A solution of AIBN (30 $\mathrm{mg}, 0.19 \mathrm{mmol})$ and tributyltin hydride $(3.2 \mathrm{~mL}, 11.24 \mathrm{mmol})$ in toluene $(125 \mathrm{~mL})$ was added dropwise over $30 \mathrm{~min}$ to a solution of the phenylselanyl esters 6 and $7(2.51 \mathrm{~g}, 7.49 \mathrm{mmol})$ in toluene $(125 \mathrm{~mL})$ at reflux under nitrogen and the mixture stirred under reflux for $36 \mathrm{~h}$. After cooling, the reaction mixture was added to aqueous potassium fluoride $(250 \mathrm{~mL})$ at $0{ }^{\circ} \mathrm{C}$ and the biphasic mixture stirred for $30 \mathrm{~min}$. The aqueous layer was extracted with ether $(250 \mathrm{~mL})$ and 
the combined organic layers dried $\left(\mathrm{MgSO}_{4}\right)$, filtered and concentrated under reduced pressure. Chromatography of the residue using light petroleum and ether $(2: 1)$ as eluent gave the title compound 8 (1.08 g, $7.1 \mathrm{mmol}, 95 \%$ ) as a clear oil (Found: $\mathrm{M}^{+}+\mathrm{NH}_{4}, 170.1176 . \mathrm{C}_{9} \mathrm{H}_{16} \mathrm{NO}_{2}$ requires $M, 170.1176)$; $\mathrm{v}_{\max } 3021,1765,1484,1457,1374,1329,1300,1208,1151,1086,1037$, $1011,986,901,836,771,741$ and 685; $\delta_{\mathrm{H}}\left(500 \mathrm{MHz}, \mathrm{CDCl}_{3}\right) 5.69(1 \mathrm{H}, \mathrm{dd}, J 5,12,4-\mathrm{H}), 5.51$ (1 H, ddd, $J$ 2.5, 4, 12, 5-H), 4.40 and 3.85 (each $1 \mathrm{H}, \mathrm{t}, J$ 8.5, 7-H), 3.00 (1 H, m, 6-H), 2.64 (1 $\mathrm{H}$, ddd, $J 5,7,13,1-\mathrm{H}), 2.19(1 \mathrm{H}, \mathrm{m}, 3-\mathrm{H}), 1.97$ and 1.29 (each $1 \mathrm{H}, \mathrm{m}, 2-\mathrm{H})$ and $1.00(3 \mathrm{H}, \mathrm{d}, J$ 7, 3- $\left.\mathrm{CH}_{3}\right) ; \delta_{\mathrm{C}}\left(75 \mathrm{MHz}, \mathrm{CDCl}_{3}\right)$ 179.6, 136.7, 122.8, 72.2, 38.4, 35.2, 29.2, 29.0, 21.4; m/z (CI) $170\left(\mathrm{M}^{+}+18,100 \%\right)$.

(1RS,3RS,6SR)-1-Hydroxy-3-methyl-9-oxo-8-oxabicyclo[4.3.0]non-4-ene $\quad(9){ }^{2 b}$ Potassium hexamethyldisilylazide $(0.5 \mathrm{M}$ in toluene, $4.2 \mathrm{~mL}, 2.11 \mathrm{mmol})$ was added to the lactone 8 (230 $\mathrm{mg}, 1.51 \mathrm{mmol})$ in tetrahydrofuran $(15 \mathrm{~mL})$ at $-78{ }^{\circ} \mathrm{C}$ under nitrogen and the solution stirred at $78{ }^{\circ} \mathrm{C}$ for $10 \mathrm{~min}$ and at $-20{ }^{\circ} \mathrm{C}$ for $30 \mathrm{~min}$. After re-cooling to $-78{ }^{\circ} \mathrm{C}$, the 2 -sulfonyloxaziridine $(423 \mathrm{mg}, 1.62 \mathrm{mmol})$ in tetrahydrofuran $(3 \mathrm{~mL})$ was added and the mixture stirred for $3 \mathrm{~h}$ at -78 ${ }^{\circ} \mathrm{C}$. After being allowed to warm to $0{ }^{\circ} \mathrm{C}$, saturated aqueous ammonium chloride $(20 \mathrm{~mL})$ was added, and the aqueous layer acidified to $\mathrm{pH} 4$ with aqueous hydrogen chloride (1 M, $2.3 \mathrm{~mL}$ ). After extraction with ether $(3 \times 25 \mathrm{~mL})$, the combined organic layers were dried $\left(\mathrm{Na}_{2} \mathrm{SO}_{4}\right)$ and concentrated under reduced presure. Chromatography of the residue using light petroleum and ether (gradient ellution up to $1: 2$ ) gave the title compound 9 (177 $\mathrm{mg}, 1.05 \mathrm{mmol}, 70 \%)$, as a white crystalline solid, m.p: $72.9-74.0{ }^{\circ} \mathrm{C}$ (Found: $\mathrm{C}$, 63.75; $\mathrm{H}, 7.0 \%$. $\mathrm{C}_{9} \mathrm{H}_{12} \mathrm{O}_{3}$ requires $\mathrm{C}, 64.3$; $\mathrm{H}, 7.2 \%$; Found: $\mathrm{M}^{+}+\mathrm{NH}_{4}, 186.1129 . \mathrm{C}_{9} \mathrm{H}_{16} \mathrm{NO}_{3}$ requires $\left.M, 186.1125\right) ; \mathrm{v}_{\max } 3427,1769,1331$, $1221,1146,1105,1012,975$ and $748 \mathrm{~cm}^{-1} ; \delta_{\mathrm{H}}\left(500 \mathrm{MHz}, \mathrm{CDCl}_{3}\right) 5.73(1 \mathrm{H}, \mathrm{dd}, J 1,10,4-\mathrm{H})$, 5.52 (1 H, ddd, J 2.5, 4, 10, 5-H), 4.45 (1 H, t, J 9, 7-H), 3.65 (1H, dd, J 9, 10.5, 7-H'), 2.91 (1 H, m, 6-H), 2.59 (1 H, br. s, OH), 2.49 (1 H, m, 3-H), 1.79 (1 H, ddd, J 1, 5, 14, 2-H), 1.26 (1 H, $\left.\mathrm{dd}, J 11.5,14,2-\mathrm{H}^{\prime}\right)$ and $1.02\left(3 \mathrm{H}, \mathrm{d}, J 7,3-\mathrm{CH}_{3}\right) ; \delta_{\mathrm{C}}\left(75 \mathrm{MHz}, \mathrm{CDCl}_{3}\right), 179.8,136.5,121.0$, 72.9, 70.8, 42.3, 36.1, 52.9 and 20.9; $m / z(\mathrm{CI}) 186\left(\mathrm{M}^{+}+18,100 \%\right)$.

\section{Benzyl (1RS,2SR,5RS)-2-benzyloxymethyl-1-hydroxy-5-methylcyclohex-3-ene-1-carb-}

oxylate (10). ${ }^{2 \mathrm{~b}}$ Benzyl bromide $(6.14 \mathrm{~mL}, 52.36 \mathrm{mmol})$ was added to a suspension of lactone 9 $(2.2 \mathrm{~g}, 13.09 \mathrm{mmol})$ and potassium hydroxide $(3.16 \mathrm{~g}, 56.3 \mathrm{mmol})$ in toluene $(20 \mathrm{~mL})$ under nitrogen. The mixture was stirred under reflux for $48 \mathrm{~h}$, cooled to room temperature, diluted with ethyl acetate $(30 \mathrm{~mL})$, washed with water $(50 \mathrm{~mL})$, dried $\left(\mathrm{Na}_{2} \mathrm{SO}_{4}\right)$ and concentrated under reduced pressure. Chromatography using light petroleum and ether $(70: 30)$ as eluent gave the title compound $\mathbf{1 0}$ (4.0 g, $10.92 \mathrm{mmol}, 83 \%$ ) as a solid, m.p. 63-63.7 ${ }^{\circ} \mathrm{C}$ (Found: $\mathrm{M}^{+}+\mathrm{NH}_{4}$, 384.2177. $\mathrm{C}_{23} \mathrm{H}_{30} \mathrm{NO}_{4}$ requires $\left.M, 384.2169\right)$; $\mathrm{v}_{\max } 3497,3030,1728,1497,1454,1223,1162$, $1117,1026,737$ and $698 \mathrm{~cm}^{-1} ; \delta_{\mathrm{H}}\left(500 \mathrm{MHz} \mathrm{CDCl}_{3}\right) 7.15(10 \mathrm{H}, \mathrm{m}, \mathrm{ArH}), 5.58(1 \mathrm{H}, \mathrm{dd}, J 1.5$, 10, 4-H), 5.37 (1 H, ddd, J 2.5, 4.5, 10, 3-H), 4.92 and 4.79 (each $1 \mathrm{H}, \mathrm{d}, J 12.5, \mathrm{PhHCH}), 4.21$ $\left(2 \mathrm{H}, \mathrm{s}, \mathrm{PhCH}_{2}\right), 3.26(1 \mathrm{H}, \mathrm{dd}, J 3.5,10,2-\mathrm{CH}), 3.19$ (1 H, dd, $J$ 9, 10, 2-CH'), 2.93 ( $1 \mathrm{H}$, br. s, $\mathrm{OH}), 2.43$ (1 H, m, 2-H), 2.29 (1 H, m, 5-H), 1.79 (1 H, dd, J 5.5, 13.5, 6- $\left.\mathrm{H}_{\mathrm{eq}}\right), 1.55$ (1 H, dd, $J$ $\left.11,13.5,6-\mathrm{H}_{\mathrm{ax}}\right)$ and $0.91\left(3 \mathrm{H}, \mathrm{d}, J 7,5-\mathrm{CH}_{3}\right) ; \delta_{\mathrm{C}}\left(75 \mathrm{MHz}, \mathrm{CDCl}_{3}\right) 175.6 .138 .2,135.8,135.3$, 
$128.8,128.6,128.5,128.4,128.0,127.9,123.2,74.7,73.1,71.1,67.6,47.4,35.2,27.5$ and 21.1; $\mathrm{m} / z(\mathrm{CI}) 384\left(\mathrm{M}^{+}+18,100 \%\right)$ and $367\left(\mathrm{M}^{+}+1,97\right)$.

(1RS,2SR,5RS)-2-Benzyloxymethyl-1-hydroxy-5-methylcyclohex-3-ene-1-carboxylic acid (11). Sodium hydroxide $(3.3 \mathrm{~g}, 82.5 \mathrm{mmol})$ in water $(22 \mathrm{~mL})$ was added to the benzyl ester 10 $(4.0 \mathrm{~g}, 11.13 \mathrm{mmol})$ in acetonitrile $(110 \mathrm{~mL})$ and the solution stirred under reflux for $24 \mathrm{~h}$. After concentration under reduced pressure, the aqueous residue was washed with ether, cooled to $0{ }^{\circ} \mathrm{C}$ and diluted to $50 \mathrm{~mL}$ with water. Aqueous hydrogen chloride $(1 \mathrm{M})$ added until the $\mathrm{pH}$ was between 1 and 2. The solution was then extracted with dichloromethane $(3 \mathrm{x} 50 \mathrm{~mL})$ and the extracts dried $\left(\mathrm{Na}_{2} \mathrm{SO}_{4}\right)$ and concentrated under reduced pressure. Recrystallization of the residue from toluene and methanol (50:1) gave the title compound 11 (1.80 g, $6.54 \mathrm{mmol}, 59 \%)$ as white crystals, m.p: $117.9-120.1{ }^{\circ} \mathrm{C}$ (Found : $\mathrm{M}^{+}+\mathrm{NH}_{4}, 294.1711 . \mathrm{C}_{16} \mathrm{H}_{24} \mathrm{NO}_{4}$ requires $M$, 294.1700); $\mathrm{v}_{\max } 3524,1679,1453,1320,1261,1174,1113,1084,1047,756,730,695$ and 642 $\mathrm{cm}^{-1} ; \delta_{\mathrm{H}}\left(500 \mathrm{MHz}, \mathrm{CDCl}_{3}\right), 7.23(5 \mathrm{H}, \mathrm{m}, \mathrm{ArH}), 5.65$ (1 H, dd, $J$ 10, 1, 4-H), 5.43 (1 H, ddd, $J$ 10, 4, 2.5, 3-H), 4.40 and 4.32 (each $1 \mathrm{H}, \mathrm{d}, J 12, \mathrm{PhHCH}), 3.42$ (1 H, dd, $J$ 4, 10, 2-CH), 3.33 (1 $\mathrm{H}$, dd, $J$ 8.5, 10, 2-CH'), 2.47 (1 H, m, 2-H), 2.35 (1 H, m, 5-H), $1.77\left(1 \mathrm{H}, \mathrm{dd}, J 14,6,6-\mathrm{H}_{\mathrm{eq}}\right)$, $1.58\left(1 \mathrm{H}, \mathrm{dd}, J 14,10.5,6-\mathrm{H}_{\mathrm{ax}}\right)$ and $0.98\left(3 \mathrm{H}, \mathrm{d}, J 7,5-\mathrm{CH}_{3}\right) ; \delta_{\mathrm{C}}\left(75 \mathrm{MHz}, \mathrm{CDCl}_{3}\right) 178.0,138.5$, 135.3, 128.8, 128.2, 128.0, 123.2, 74.9, 73.4, 71.3, 46.8, 36.2, 27.5 and 21.0; $\mathrm{m} / \mathrm{z}$ (CI) 294 $\left(\mathrm{M}^{+}+18,10 \%\right)$ and $276\left(\mathrm{M}^{+}, 50\right)$.

Methyl (1RS,2SR,5RS)-2-benzyloxymethyl-1-hydroxy-5-methylcyclohex-3-ene-1-carboxylate (12). Trimethylsilyldiazomethane $(0.8 \mathrm{~mL}, 1.6 \mathrm{mmol})$ was added to the acid 11 (139 $\mathrm{mg}$, $0.5 \mathrm{mmol})$ in toluene $(1.9 \mathrm{~mL})$ and methanol $(1.3 \mathrm{~mL})$ at $0{ }^{\circ} \mathrm{C}$ under nitrogen until the yellow colour of the trimethylsilyldiazomethane persisted. The yellow solution was then stirred at $0{ }^{\circ} \mathrm{C}$ for $1 \mathrm{~h}$, acetic acid $(0.2 \mathrm{~mL})$ was added and the mixture stirred for $10 \mathrm{~min}$. After concentration under reduced pressure, the residue was chromatographed using light petroleum and ether $(1: 1)$ as eluent to give the title compound $12(130 \mathrm{mg}, 0.45 \mathrm{mmol}, 90 \%)$ as a white solid, m.p. 68.2$70.1{ }^{\circ} \mathrm{C}$ (Found: $\mathrm{M}^{+}+\mathrm{NH}_{4}, 308.1859 . \mathrm{C}_{17} \mathrm{H}_{26} \mathrm{NO}_{4}$ requires $M, 308.1856$ ); $\mathrm{v}_{\max } 3492,1731,1454$, $1258,1164,1119,1080,1028,736$ and $698 \mathrm{~cm}^{-1} ; \delta_{\mathrm{H}}\left(500 \mathrm{MHz}, \mathrm{CDCl}_{3}\right) 7.20(5 \mathrm{H}, \mathrm{m}, \mathrm{ArH}), 5.63$ (1 H, dd, $J 1,10,4-\mathrm{H}), 5.40$ (1 H, ddd, $J 2.5,4.5,10,3-\mathrm{H}), 4.34$ and 4.31 (each $1 \mathrm{H}, \mathrm{d}, J 12$, $\mathrm{PhHCH}), 3.54$ (3 H, s, $\left.\mathrm{OCH}_{3}\right), 3.32$ (1 H, dd, J 3, 10, 2-CH), 3.23 (1 H, dd, J 9, 10, 2-CH'), 2.99 ( $1 \mathrm{H}$, br. s, OH), 2.45 (1 H, m, 2-H), 2.37 (1 H, m, 5-H), 1.81 (1 H, dd, J 5.5, 13.5, 6-CHeq $), 1.57$ $\left(1 \mathrm{H}, \mathrm{dd}, J 11,13.5,6-\mathrm{CH}_{\mathrm{ax}}\right)$ and $0.96\left(3 \mathrm{H}, \mathrm{d}, J 7,5-\mathrm{CH}_{3}\right) ; \delta_{\mathrm{C}}\left(75 \mathrm{MHz}, \mathrm{CDCl}_{3}\right), 176.2,138.1$, $135.3,128.6,127.8,123.1,74.6,73.2,71.5,52.8,47.3,35.3,27.4$ and $21.1 ; m / z(\mathrm{CI}) 291\left(\mathrm{M}^{+}+1\right.$, $100 \%)$ and $308(35)$.

\section{(1RS,4RS,5RS,6SR,7RS)-5-Benzyloxymethyl-4,6-dihydroxy-7-methyl-2-oxabicyclo-}

[2.2.2]octan-3-one (13), (1RS,4RS,5RS,6RS,7RS)-5-benzyloxymethyl-4,6-dihydroxy-7methyl-2-oxabicyclo[2.2.2]octan-3-one (14) and (1RS,3RS,4SR,5SR,8RS)-8-benzyloxymethyl-1,4-dihydroxy-3-methyl-6-oxabicyclo[3.2.1]octan-7-one(15). meta-Chloro- peroxybenzoic acid $(190 \mathrm{mg}, 0.77 \mathrm{mmol})$ in chloroform $(0.8 \mathrm{~mL})$ was added to the carboxylic acid 11 $(178 \mathrm{mg}, 0.64 \mathrm{mmol})$ in chloroform $(4.2 \mathrm{~mL})$ and the solution stirred at room temperature for 72 $\mathrm{h}$. The reaction mixture was then washed with a saturated aqueous sodium bicarbonate and the 
aqueous layer extracted with chloroform $(5 \mathrm{~mL})$. The combined organic extracts were dried $\left(\mathrm{MgSO}_{4}\right)$ and concentrated under reduced pressure. Chromatography using light petroleum and ether (70 : 30) gave the title compound $13(28 \mathrm{mg}, 0.096 \mathrm{mmol}, 15 \%)$ as an oil (Found: $\mathrm{M}^{+}+\mathrm{NH}_{4}, 310.1651 . \mathrm{C}_{16} \mathrm{H}_{24} \mathrm{NO}_{5}$ requires $\left.M, 310.1649\right)$; $v_{\max } 3446,1752,1455,1365,1260$, 1067, 798, 741 and $698 \mathrm{~cm}^{-1} ; \delta_{\mathrm{H}}\left(500 \mathrm{MHz}, \mathrm{CDCl}_{3}\right) 7.25(5 \mathrm{H}, \mathrm{m}, \mathrm{ArH}), 4.43$ and 4.41 (each 1 H, d, J 12, PhHCH), 4.25 (1 H, d, J 3.5, 1-H), 3.73 (1 H, t, J 4, 6-H), 3.61 (1 H, dd, J 4, 9, 5$\mathrm{CH}), 3.42$ (2 H, m, 5-CH', OH), 2.46 (1 H, m, 7-H), 2.22 (1 H, dd, J 10.5, 13, 8-H), 1.94 (1 H, q, $J 4,5-\mathrm{H}), 1.25\left(1 \mathrm{H}, \mathrm{dd}, J 13,5,8-\mathrm{H}^{\prime}\right)$ and $1.03\left(3 \mathrm{H}, \mathrm{d}, J 7.5,7-\mathrm{CH}_{3}\right) ; \delta_{\mathrm{C}}\left(75 \mathrm{MHz}, \mathrm{CDCl}_{3}\right)$ $177.5,138.1,128.8,128.0,127.9,79.9,75.9,73.8,70.9,68.0,65.9,48.5,26.6$ and 19.6; $\mathrm{m} / \mathrm{z}$ (CI) $310\left(\mathrm{M}^{+}+18,100 \%\right)$ and $293\left(\mathrm{M}^{+}+1,20\right)$. The second fraction was the title compound $14(56 \mathrm{mg}$, $0.192 \mathrm{mmol}, 30 \%$ ) as a crystalline solid, m.p. 123.7-124.3 ${ }^{\circ} \mathrm{C}$ (Found: $\mathrm{M}^{+}, 292.1305 . \mathrm{C}_{16} \mathrm{H}_{20} \mathrm{O}_{5}$ requires $M, 292.1305) ; \mathrm{v}_{\max } 3434,1752,1455,1261,1108,1068,799,740$ and $699 \mathrm{~cm}^{-1} ; \delta_{\mathrm{H}}(500$ $\left.\mathrm{MHz}, \mathrm{CDCl}_{3}\right), 7.27$ (5 H, m, ArH), 4.45 and 4.43 (each $\left.1 \mathrm{H}, \mathrm{d}, J 12, \mathrm{PhHCH}\right), 4.15(1 \mathrm{H}, \mathrm{d}, J 5$, 1-H), 4.08 (1 H, t, J 5, 6-H), 3.56 (1 H, dd, J 10, 5, 5-CH), 3.45 (1 H, dd, J 10, 7, 5-CH'), 3.29 (1 $\mathrm{H}, \mathrm{s}, \mathrm{OH}), 2.52$ (1 H, m, 7-H), 2.26 (1 H, dd, J 15, 10, 8-H), 2.10 (1 H, br. s, OH), 1.99 (1 H, m, $J$ 5-H), $1.25\left(1 \mathrm{H}, \mathrm{dd}, J 15,5,8-\mathrm{H}^{\prime}\right)$ and $1.05\left(3 \mathrm{H}, \mathrm{d}, J 5,7-\mathrm{CH}_{3}\right) ; \delta_{\mathrm{C}}\left(75 \mathrm{MHz}, \mathrm{CDCl}_{3}\right) 177.3$, $137.9,128.7,128.1,127.9,82.0,73.7,70.8,69.4,68.2,49.6,40.5,26.0$ and $19.3 ; \mathrm{m} / z$ (CI) 310 $\left(\mathrm{M}^{+}+18,100 \%\right)$ and $293\left(\mathrm{M}^{+}+1,20\right)$. The third fraction was the title compound 15 (56 mg, 0.192 mmol, $30 \%$ ) as an oil (Found: $\mathrm{M}^{+}, 292.1302 . \mathrm{C}_{16} \mathrm{H}_{20} \mathrm{O}_{5}$ requires $M, 292.1305$ ); $\mathrm{v}_{\max } 3432,1774$, 1454, 1161, 1101, 1023, 963, 740 and $698 \mathrm{~cm}^{-1}: \delta_{\mathrm{H}}\left(500 \mathrm{MHz}, \mathrm{CDCl}_{3}\right) 7.29(5 \mathrm{H}, \mathrm{m}, \mathrm{ArH}), 4.50$ $(1 \mathrm{H}, \mathrm{d}, J 4,5-\mathrm{H}), 4.48$ and 4.45 (each $1 \mathrm{H}, \mathrm{d}, J 12, \mathrm{PhHCH}), 3.80(1 \mathrm{H}, \mathrm{t}, J 3,4-\mathrm{H}), 3.67$ (1 H, dd, $J$ 5.5, 10, 8-CH), 3.58 (1 H, dd, $J$ 7, 10, 8-CH'), 3.02 (1 H, br. s, OH), 2.81 (1 H, dd, J 7, 5.5, 8-H), 2.26 (1 H, dd, J 9, 13.5, 2-H), 2.08 (1 H, m, 3-H), 1.65 (1 H, dd, J 3.5, 13.5, 2-H'), 1.5 (1 $\mathrm{H}$, br. s, $\mathrm{OH})$ and $1.05\left(3 \mathrm{H}, \mathrm{d}, J 7,3-\mathrm{CH}_{3}\right) ; \delta_{\mathrm{C}}\left(75 \mathrm{MHz}, \mathrm{CDCl}_{3}\right) 179.2,137.6,128.8,128.3$, $128.0,80.8,74.4,71.4,67.3,44.5,39.8,34.5,26.3$ and $21.2 ; m / z(C I) 310\left(\mathrm{M}^{+}+18,100 \%\right)$.

(1RS,3RS,5SR,8RS)-8-Benzyloxymethyl-1-hydroxy-3-methyl-6-oxabicyclo[3.2.1]octane-4,7dione (16). Pyridinium chlorochromate $(301 \mathrm{mg}, 1.37 \mathrm{mmol})$ in dichloromethane $(4 \mathrm{~mL})$ was added to a solution of the hydroxylactone $15(200 \mathrm{mg}, 0.68 \mathrm{mmol})$ in dichloromethane $(4 \mathrm{~mL})$ and the mixture stirred for $12 \mathrm{~h}$. The reaction mixture was then washed with water, dried $\left(\mathrm{MgSO}_{4}\right)$ and concentrated under reduced pressure. Chromatography of the residue using light petroleum and ether (60:40) gave the title compound $16(168 \mathrm{mg}, 0.58 \mathrm{mmol}, 85 \%)$ as a clear oil (Found: $\mathrm{M}^{+}+\mathrm{NH}_{4}, 308.1491 . \mathrm{C}_{16} \mathrm{H}_{22} \mathrm{NO}_{5}$ requires $M, 308.1492$ ); $v_{\max } 3432,1769,1644,1333$, $1217,1145,1106,1013,976$ and $749 \mathrm{~cm}^{-1} ; \delta_{\mathrm{H}}\left(500 \mathrm{MHz}, \mathrm{CDCl}_{3}\right) 7.25(5 \mathrm{H}, \mathrm{m}, \mathrm{ArH}), 4.53(1 \mathrm{H}$, s, 5-H), 4.47 (2 H, s, $\left.\mathrm{PhCH}_{2}\right), 3.69$ (1 H, dd, J 4.5, 10, 8-CH), 3.63 (1 H, dd, J 6.5, 10, 8-CH'), 3.27 (1 H, br. s, OH), 2.75 (1 H, pent, $J$ 7, 3-H), 2.55 (1 H, dd, $J 4.5,6.5,8-\mathrm{H}), 2.34$ (1 H, dd, $J$ $7.5,13.5,2-\mathrm{H}), 1.89\left(1 \mathrm{H}, \mathrm{d}, J 13.5,2-\mathrm{H}^{\prime}\right)$ and $1.24\left(3 \mathrm{H}, \mathrm{d}, J 8,3-\mathrm{CH}_{3}\right) ; \delta_{\mathrm{C}}\left(75 \mathrm{MHz}, \mathrm{CDCl}_{3}\right)$ $204.4,177.8,137.3,128.9,128.2,127.9,82.1,74.0,73.7,66.1,49.4,39.7,39.2$ and $20.5 ; \mathrm{m} / z$ (CI) $308\left(\mathrm{M}^{+}+18,20 \%\right)$.

(1RS,3RS,4SR,5SR,8RS)-8-Benzyloxymethyl-1,4-dihydroxy-3-methyl-6-oxabicyclo-[3.2.1] octan-7-one (15) and (1RS,3RS,4RS,5SR,8RS)-8-benzyloxymethyl-1,4-dihydroxy-3-methyl- 
6-oxabicyclo[3.2.1]octan-7-one (17). Sodium borohydride ( $2.5 \mathrm{mg}, 0.066 \mathrm{mmol}$ ) was added to the ketone $16(25 \mathrm{mg}, 0.066 \mathrm{mmol})$ in ethanol $(0.3 \mathrm{~mL})$ at $0{ }^{\circ} \mathrm{C}$. The reaction mixture was stirred at $0{ }^{\circ} \mathrm{C}$ for $1 \mathrm{~h}$, water $(1 \mathrm{~mL})$ was added, the mixture was stirred for $5 \mathrm{~min}$ and then extracted with dichloromethane $(2 \times 5 \mathrm{~mL})$. The organic extracts were dried $\left(\mathrm{MgSO}_{4}\right)$ and concentrated under reduced pressure. Chromatography of the residue using light petroleum and ether $(3: 7)$ gave the alcohol 15 (7.7 $\mathrm{mg}, 0.026 \mathrm{mmol}, 40 \%$ ) and the title compound 17 (8 $\mathrm{mg}, 0.027 \mathrm{mmol}$, $41 \%$ ) as an oil (Found: $\mathrm{M}^{+}+\mathrm{H}, 293.1383 . \mathrm{C}_{16} \mathrm{H}_{21} \mathrm{O}_{5}$ requires $M, 293.1384$ ); $\mathrm{v}_{\max } 3450,1775$, $1640,1455,1162,1105,1024,964,743$ and $700 \mathrm{~cm}^{-1} ; \delta_{\mathrm{H}}\left(500 \mathrm{MHz}, \mathrm{CDCl}_{3}\right) 7.24(5 \mathrm{H}, \mathrm{m}$, ArH), 4.53 (1 H, d, J 5, 5-H), 4.59 and 4.45 (each $1 \mathrm{H}, \mathrm{d}, J$ 12, $\mathrm{PhHCH}), 3.91$ (1 H, q, J 5, 4-H), 3.67 and 3.59 (each $1 \mathrm{H}$, dd, $J 6,10,8-\mathrm{CH}), 2.80(1 \mathrm{H}, \mathrm{t}, J 6,8-\mathrm{H}), 1.87(1 \mathrm{H}, \mathrm{m}, 3-\mathrm{H}), 1.68$ (2 $\left.\mathrm{H}, \mathrm{m}, 2-\mathrm{H}_{2}\right)$ and $0.98\left(3 \mathrm{H}, \mathrm{d}, J 7,3-\mathrm{CH}_{3}\right) ; \mathrm{m} / z(\mathrm{CI}) 310\left(\mathrm{M}^{+}+18,100 \%\right)$.

Methyl (1RS,2RS,3RS,5RS,6SR)-2-benzyloxymethyl-3-hydroxy-5-methyl-7-oxabicyclo[4.1.0]heptane-3-carboxylate (18) and methyl (1SR,2RS,3RS,5RS,6RS)-2-benzyloxymethyl-3-hydroxy-5-methyl-7-oxabicyclo[4.1.0]heptane-3-carboxylate (19). metaChloroperoxybenzoic acid $(930 \mathrm{mg}, 3.77 \mathrm{mmol})$ in chloroform $(30 \mathrm{~mL})$ was added to the methyl ester $12(910 \mathrm{mg}, 3.14 \mathrm{mmol})$ in chloroform $(30 \mathrm{~mL})$ at room temperature and the mixture stirred at room temperature for $18 \mathrm{~h}$. Saturated aqueous sodium bicarbonate $(10 \mathrm{~mL})$ was added and the aqueous phase washed with dichloromethane $(2 \times 10 \mathrm{~mL})$. The organic extracts were dried $\left(\mathrm{MgSO}_{4}\right)$ and concentrated under reduced pressure. Chromatography of the residue using dichloromethane and ether (4:1) gave the title compound 18 (200 mg, $0.65 \mathrm{mmol}, 20 \%)$ (Found: $\mathrm{M}^{+}+\mathrm{NH}_{4}, 324.1809 . \mathrm{C}_{17} \mathrm{H}_{26} \mathrm{NO}_{5}$ requires $M, 324.1805$ ); $\mathrm{v}_{\max } 3451,1738,1455,1366$, 1252, 1108, 804, 739 and $700 \mathrm{~cm}^{-1} ; \delta_{\mathrm{H}}\left(500 \mathrm{MHz}, \mathrm{CDCl}_{3}\right) 7.26(5 \mathrm{H}, \mathrm{m}, \mathrm{ArH}), 4.41$ and 4.37 (each $1 \mathrm{H}, \mathrm{d}, J 12, \mathrm{PhHCH}), 3.59\left(3 \mathrm{H}, \mathrm{s}, \mathrm{OCH}_{3}\right), 3.40\left(2 \mathrm{H}, \mathrm{m}, 2-\mathrm{CH}_{2}\right), 3.37(1 \mathrm{H}, \mathrm{dd}, J 1,4,1-$ $\mathrm{H}$ or 6-H), 2.97 (1 H, d, J 4, 1-H or 6-H), $2.65(1 \mathrm{H}, \mathrm{t}, J 6,2-\mathrm{H}), 2.30(1 \mathrm{H}, \mathrm{m}, 5-\mathrm{H}), 1.88(1 \mathrm{H}$, $\left.\mathrm{dd}, J 7,14,4-\mathrm{H}_{\mathrm{eq}}\right), 1.53\left(1 \mathrm{H}, \mathrm{dd}, J 10,14,4-\mathrm{H}_{\mathrm{ax}}\right), 1.50(1 \mathrm{H}, \mathrm{br} . \mathrm{s}, \mathrm{OH})$ and $1.05(3 \mathrm{H}, \mathrm{d}, J 7.5$, $\left.5-\mathrm{CH}_{3}\right) ; \delta_{\mathrm{C}}\left(75 \mathrm{MHz}, \mathrm{CDCl}_{3}\right)$ 19.5, 26.0, 35.6, 42.6, 52.6, 57.4, 57.8, 68.6, 73.7, 75.8, 128.6, 128.6, 128.7, 137.8 and 173.3; $\mathrm{m} / \mathrm{z}$ (CI) $324\left(\mathrm{M}^{+}+18,30 \%\right)$ and $307\left(\mathrm{M}^{+}+1,100\right)$. The second fraction was the title compound 19 (600 mg, $1.96 \mathrm{mmol}, 62 \%$ ) (Found: $\mathrm{M}^{+}+\mathrm{NH}_{4}, 324.1805$. $\mathrm{C}_{17} \mathrm{H}_{26} \mathrm{NO}_{5}$ requires $\left.M, 324.1805\right) ; \mathrm{v}_{\max } 3477,1733,1455,1364,1258,1167,1112,1025,778$, 740 and $700 \mathrm{~cm}^{-1} ; \delta_{\mathrm{H}}\left(500 \mathrm{MHz}, \mathrm{CDCl}_{3}\right) 7.20(5 \mathrm{H}, \mathrm{m}, \mathrm{ArH}), 4.39\left(2 \mathrm{H}, \mathrm{s}, \mathrm{PhCH}_{2}\right), 3.61(1 \mathrm{H}$, dd, $J 3.5,10,2-\mathrm{CH}), 3.51$ ( $\left.3 \mathrm{H}, \mathrm{s}, \mathrm{OCH}_{3}\right), 3.36$ (1 H, dd, $\left.J 9,10,2-\mathrm{CH}^{\prime}\right), 3.25$ (1 H, dd, $J 4,5,1-$ H or 6-H), 3.09 (1 H, d, J 4, 1-H or 6-H), 3.01 (1 H, br. s, OH), 2.26 (2 H, m, 2-H, 5-H), 1.56 (1 $\left.\mathrm{H}, \mathrm{dd}, J 13,14,4-\mathrm{H}_{\mathrm{ax}}\right), 1.40\left(1 \mathrm{H}, \mathrm{ddd}, J 1,5,14,4-\mathrm{H}_{\mathrm{eq}}\right)$ and $1.05\left(3 \mathrm{H}, \mathrm{d}, J 7,5-\mathrm{CH}_{3}\right) ; \delta_{\mathrm{C}}(75$ $\left.\mathrm{MHz}, \mathrm{CDCl}_{3}\right)$ 176.1, 138.2, 128.7, 128.1, 127.9, 73.6, 73.4, 68.5, 57.2, 53.7, 53.1, 44.9, 31.9, 26.0 and $18.4 ; \mathrm{m} / \mathrm{z}(\mathrm{CI}) 324\left(\mathrm{M}^{+}+18,90 \%\right)$ and $307\left(\mathrm{M}^{+}+1,40\right)$.

Vanadyl acetylacetonate $(5 \mathrm{mg}, 0.016 \mathrm{mmol})$ in benzene $(0.3 \mathrm{~mL})$ was added to the methyl ester 12 (329 mg, $1.13 \mathrm{mmol})$ in benzene $(1 \mathrm{~mL})$. The brown solution was stirred under reflux and tert-butyl hydrogen peroxide $(0.37 \mathrm{~mL}, 1.85 \mathrm{mmol})$ was added. The reaction mixture was stirred under reflux for $4.5 \mathrm{~h}$ then cooled to room temperature and washed with aqueous sodium sulphite $(10 \%)$ and brine. The organic extracts were dried $\left(\mathrm{MgSO}_{4}\right)$ and concentrated under reduced 
pressure. Chromatography of the residue using dichloromethane and ether (4:1) as eluent gave the cis-hydroxyepoxide 18 (280 mg, $0.91 \mathrm{mmol}, 81 \%$ ) as a colourless oil with spectroscopic data identical to those reported above.

Benzyl (1RS,2RS,3RS,5RS,6SR)-2-benzyloxymethyl-3-hydroxy-5-methyl-7-oxabicyclo[4.1.0]heptane-3-carboxylate (20) and benzyl (1SR,2RS,3RS,5RS,6RS)-2-benzyloxy-methyl3-hydroxy-5-methyl-7-oxabicyclo[4.1.0]heptane-3-carboxylate $\quad(21){ }^{2 b}$ metaChloroperoxybenzoic acid in dichloromethane $(1 \mathrm{M}, 7.4 \mathrm{~mL})$ was added to the benzyl ester 10 $(1.36 \mathrm{~g}, 3.71 \mathrm{mmol})$ in dichloromethane at $0{ }^{\circ} \mathrm{C}$ and the reaction mixture warmed to room temperature and stirred for $5 \mathrm{~h}$. The mixture was then washed with aqueous sodium sulfite (10 $\%)$, dried $\left(\mathrm{MgSO}_{4}\right)$ and concentrated under reduced pressure. Chromatography of the residue using light petroleum and ether (3:2) gave a mixture of the title compounds 20 and 21 (1.2 g, $3.14 \mathrm{mmol}, 85 \% ; 20: 21=33: 67$ ) as a colourless oil (Found: $\mathrm{M}^{+}+\mathrm{NH}_{4}, 400.2117 . \mathrm{C}_{23} \mathrm{H}_{30} \mathrm{NO}_{5}$ requires $M, 400.2118)$; $\mathrm{v}_{\max } 3471,3031,1734,1497,1454,1365,1219,1164,1108,1024,737$ and $698 \mathrm{~cm}^{-1} ; \delta_{\mathrm{H}}\left(500 \mathrm{MHz}, \mathrm{CDCl}_{3}\right)($ cis-isomer 20) $7.35(10 \mathrm{H}, \mathrm{m}, \mathrm{ArH}), 5.20$ and 5.15 (each 1 $\mathrm{H}, \mathrm{d}, J 12.5, \mathrm{CO}_{2} \mathrm{CHHPh}$ ), 4.38 and 4.34 (each $\left.1 \mathrm{H}, \mathrm{d}, J 14.5, \mathrm{OCH} H \mathrm{Ph}\right), 3.50\left(2 \mathrm{H}, \mathrm{m}, 2-\mathrm{CH}_{2}\right)$, $3.39(1 \mathrm{H}, \mathrm{dd}, J 1,4,1-\mathrm{H}$ or 6-H), $3.09(1 \mathrm{H}, \mathrm{d}, J 4,1-\mathrm{H}$ or $6-\mathrm{H}), 2.80(1 \mathrm{H}, \mathrm{m}, 2-\mathrm{H}), 2.42(1 \mathrm{H}$, m, 5-H), 2.04 (1 H, dd, J 7, 14, 4-H), $1.68\left(1 \mathrm{H}, \mathrm{dd}, J 10,14,4-\mathrm{H}^{\prime}\right)$ and $1.18\left(3 \mathrm{H}, \mathrm{d}, J 7,5-\mathrm{CH}_{3}\right)$; (trans-isomer 21) $7.35(10 \mathrm{H}, \mathrm{m}, \mathrm{ArH}), 5.12$ and 4.97 (each $\left.1 \mathrm{H}, \mathrm{d}, J 12.5, \mathrm{CO}_{2} \mathrm{CH} \mathrm{HPh}\right), 4.45$ and 4.41 (each $1 \mathrm{H}, \mathrm{d}, J 12, \mathrm{OCHHPh}), 3.72(1 \mathrm{H}, \mathrm{dd}, J 5,10,2-\mathrm{CH}), 3.45(1 \mathrm{H}, \mathrm{dd}, J 10,9,2-$ $\left.\mathrm{CH}^{\prime}\right), 3.33$ (1 H, t, J 4, 1-H or 6-H), 3.15 (1 H, d, J 4, 1-H or 6-H), 3.09 (1 H, br. s, OH), 2.43 (2 $\mathrm{H}, \mathrm{m}, 5-\mathrm{H}, 2-\mathrm{H}), 1.73$ (1 H, t, $J$ 14, 4-H), 1.59 (1 H, dd, $J$ 5, 14, 4-H') and 1.19 (3 H, d, $J$ 7, 5$\left.\mathrm{CH}_{3}\right) ; m / z(\mathrm{CI}) 400\left(\mathrm{M}^{+}+18,30 \%\right)$ and $383\left(\mathrm{M}^{+}+1,20\right)$.

(1RS,4RS,5RS,6RS,7RS)-5-Benzyloxymethyl-4,6-dihydroxy-7-methyl-2-oxabicyclo-[2.2.2] octan-3-one (14) and methyl (1RS,2RS,3SR,4SR,5RS)-2-benzyloxymethyl-1,3,4-trihydroxy5-methylcyclohexane-1-carboxylate (22). Sulfuric acid (0.2 M; $2 \mathrm{~mL}, 0.39 \mathrm{mmol})$ was added to the cis-hydroxyepoxide $18(100 \mathrm{mg}, 0.33 \mathrm{mmol})$ in acetonitrile $(3.3 \mathrm{~mL})$ and the reaction mixture stirred under reflux for $48 \mathrm{~h}$. The mixture was then cooled to room temperature and concentrated under reduced pressure. The residue was dissolved in dichloromethane $(10 \mathrm{~mL})$ and the solution washed with water $(10 \mathrm{~mL})$, dried $\left(\mathrm{MgSO}_{4}\right)$ and the solvent removed under reduced pressure. Chromatography of the residue using light petroleum and ether $(40: 60)$ with gradient elution to neat ether gave the lactone 14 (64 mg, $0.2 \mathrm{mmol}, 61 \%)$ followed by the title compound 22 (36 mg, $0.11 \mathrm{mmol}, 33 \%$ ) as a white solid, m.p. 97.3-100 ${ }^{\circ} \mathrm{C}$ (Found: $\mathrm{M}^{+}+\mathrm{H}$, 325.1641. $\mathrm{C}_{17} \mathrm{H}_{25} \mathrm{O}_{6}$, requires $\left.M, 325.1646\right)$; $\mathrm{v}_{\max } 3411,1727,1641,1454,1367,1264,1062$, 1029 and $739 \mathrm{~cm}^{-1}$; $\delta_{\mathrm{H}}\left(500 \mathrm{MHz}, \mathrm{CDCl}_{3}\right) 7.23(5 \mathrm{H}, \mathrm{m}, \mathrm{ArH}), 4.36\left(2 \mathrm{H}, \mathrm{s}, \mathrm{PhCH}_{2}\right), 3.98(1 \mathrm{H}$, m, 3-H), 3.71 (1 H, dd, $J 10,6,2-\mathrm{CH}), 3.56\left(3 \mathrm{H}, \mathrm{s}, \mathrm{OCH}_{3}\right), 3.36$ (1 H, dd, J 10, 7, 2-CH'), 3.09 $(1 \mathrm{H}, \mathrm{t}, J 10,4-\mathrm{H}), 3.05$ and 2.83 (each $1 \mathrm{H}$, br. s, OH), $2.39(1 \mathrm{H}, \mathrm{m}, 2-\mathrm{H}), 2.28(1 \mathrm{H}$, br. s, $\mathrm{OH}), 1.77$ (1 H, m, 5-H), 1.76 (1 H, t, J 12.5, 6-H), 1.64 (1 H, dd, J 10, 2, 6-H') and 0.99 (3 H, d, $J$ 6, 5- $\left.\mathrm{CH}_{3}\right) ; \delta_{\mathrm{C}}\left(75 \mathrm{MHz}, \mathrm{CDCl}_{3}\right) 175.1,137.8,128.7,128.1,127.9$, 76.5, 76.0, 73.6, 72.9, 67.2, 52.9, 50.0, 36.7, 32.7 and 18.1; $\mathrm{m} / \mathrm{z}(\mathrm{CI}) 342\left(\mathrm{M}^{+}+18,100 \%\right), 325(50)$ and $307(30)$. 
Following the above procedure, the trans-hydroxyepoxide $19(418 \mathrm{mg}, 1.3 \mathrm{mmol})$ gave the lactone 14 (350 mg, $1.2 \mathrm{mmol}, 88 \%$ ) isolated from the crude product mixture by crystallisation.

(1RS,4RS,5RS,6RS,7RS)-5-Benzyloxymethyl-4,6-dihydroxy-7-methyl-2-oxabicyclo[2.2.2]octan-3-one (14) and benzyl (1RS,2RS,3SR,4SR,5RS)-2-benzyloxymethyl-1,3,4trihydroxy-5-methylcyclohexane-1-carboxylate (23). Aqueous sulfuric acid (18 mL, 3.6 mmol, $0.2 \mathrm{M})$ was added to a mixture of the epoxy esters 20 and $21(20: 21=1: 2 ; 1.15 \mathrm{~g}, 3.01$ $\mathrm{mmol})$ in acetonitrile $(30 \mathrm{~mL})$ and the solution stirred under reflux for $48 \mathrm{~h}$. The reaction mixture was then cooled to room temperature and concentrated under reduced pressure. The residue was taken up in dichloromethane and the solution washed with brine, dried $\left(\mathrm{MgSO}_{4}\right)$ and concentrated under reduced pressure. Chromatography of the residue using light petroleum and ether (1 : 4) gave the lactone $14(620 \mathrm{mg}, 2.1 \mathrm{mmol}, 70 \%)$ followed by the title compound 23 (361 mg, $0.9 \mathrm{mmol}, 30 \%$ ) as a white crystalline solid, m.p 127.7-129 ${ }^{\circ} \mathrm{C}$ (Found: $\mathrm{M}^{+}+\mathrm{NH}_{4}$, 418.2219. $\mathrm{C}_{23} \mathrm{H}_{32} \mathrm{NO}_{6}$ requires $\left.M, 418.2224\right)$; $\mathrm{v}_{\max } 3400,1727,1455,1247,1061,1028,737$ and $697 \mathrm{~cm}^{-1} ; \delta_{\mathrm{H}}\left(500 \mathrm{MHz}, \mathrm{CDCl}_{3}\right) 7.28(10 \mathrm{H}, \mathrm{m}, \mathrm{ArH}), 5.05$ and 4.96 (each $1 \mathrm{H}, \mathrm{d}, J 12$, $\mathrm{CO}_{2} \mathrm{CHHPh}$ ), 4.32 and 4.30 (each $1 \mathrm{H}, \mathrm{d}, J$ 12, OCHHPh), $4.04(1 \mathrm{H}, \mathrm{dd}, J 5,10,3-\mathrm{H}), 3.73$ (1 $\mathrm{H}, \mathrm{dd}, J 6,10,2-\mathrm{CH}), 3.65$ (2 H, br. s. 2 x OH), 3.38 (1 H, dd, $\left.J 10,8,2-\mathrm{CH}^{\prime}\right), 3.15$ (1 H, t, $J 10$, 4-H), 2.50 (1 H, m, 2-H), $1.86-1.73\left(3 \mathrm{H}, \mathrm{m}, 5-\mathrm{H}, 6-\mathrm{H}_{2}\right)$ and $1.04\left(3 \mathrm{H}, \mathrm{d}, J 5.5,5-\mathrm{CH}_{3}\right) ; \delta_{\mathrm{C}}(75$ $\left.\mathrm{MHz} \mathrm{CDCl}_{3}\right)$ 174.4, 138.1, 135.6, 128.8, 128.5, 128.6, 128.0, 76.4, 76.1, 73.4, 72.2, 67.6, 66.8, $50.2,36.4,32.6$ and 18.3; $m / z(\mathrm{CI}) 418\left(\mathrm{M}^{+}+18,80 \%\right)$.

Crystal data for lactone (14). $\mathrm{C}_{16} \mathrm{H}_{20} \mathrm{O}_{5}, M=292.32$, monoclinic, $a=11.755(3), b=11.749(3)$, $c=11.929(3) \AA, \beta=116.464(3)^{\circ}, U=1474.8(5) \AA^{3}, T=100 \mathrm{~K}$, space group $P 2_{1}$ (no. 4$), Z=4$, $\mu(\mathrm{Mo}-\mathrm{K} \alpha)=0.097 \mathrm{~mm}^{-1}, 9487$ reflections measured, 2732 unique $(R \operatorname{int}=0.089)$ which were used in all calculations. The final $R(F)$ was 0.05238 using 1739 with $\mathrm{I}>2 \sigma(\mathrm{I})$, wR2 $=0.11801$ (all data). Data have been deposited with the Cambridge Crystallographic Data Centre, CCDC number 617539 .

\section{Acknowledgements}

We thank the EPSRC for a studentship (to C.V.).

\section{References and Notes}

1. Bister, B.; Bischoff, D.; Strobele, M.; Riedlinger, J.; Reicke, A.; Wolter, F.; Bull, A. T.; Zahner, H.; Fiedler, H-P.; Sussmuth, R. D. Angew. Chem. Int. Edn. 2004, 43, 2574.

2. (a) Rath, J.-P.; Eipert, M.; Kinast, S.; Maier, E. Synlett 2005, 314. (b) Rath, J.-P.; Kinast, S.; Maier, M. E. Org. Lett. 2005, 7, 3089. (c) Zografos, A. L.; Yiotakis, A.; Georgiadis, D. Org. Lett. 2005, 7, 4515. (d) Snider, B.; Zou, Y. Org. Lett. 2005, 7, 4939. (e) Couladourros, E. A.; Bouzas, E. A.; Magos, A. D. Tetrahedron 2006, 62, 5272. 
3. (a) Zapf, C. W.; Harrison, B. A.; Drahl, C.; Sorensen, E. J. Angew. Chem. Int. Edn. Engl. 2005, 44, 6533. (b) Nicolaou, K. C.; Harrison, S. T. Angew. Chem. Int. Edn. Engl. 2006, 45, 3256.

4. The metathesis approach was used by Nicolaou and Harrison in their synthesis of the abyssomicins (see ref. 3).

5. (a) House, H. O.; Cronin, T. H. J. Org. Chem. 1965, 30, 1061. (b) Lee, H. W.; Lee, W. B.; Lee, I.-Y. C. Bull. Korean Chem. Soc. 1994, 15, 448. (c) Cayzer, T. N.; Paddon-Row, M. N. Moran, D.; Payne, A. D.; Sherburn, M. S.; Turner, P. J. Org. Chem. 2005, 70, 5561. (d) Corey, E. J.; Petrzilka, M. Tetrahedron Lett. 1975, 16, 2537.

6. Ward, D. E.; Abaee, M. S. Org. Lett. 2000, 2, 3937.

7. Brettle, R.; Cummings, D. P. J. Chem. Soc. Perkin Trans. I 1977, 2385.

8. Stojanovic, A.; Renaud, P. Synlett 1997, 181.

9. Davis, F. A.; Stringer, O. D. J. Org. Chem. 1982, 47, 1774.

10. Lafontaine, J. A.; Leahy, J. W. Tetrahedron Lett. 1995, 36, 6029. 